\title{
PRODUTIVIDADE DA CULTURA DA PINHA (Annona squamosa L.) EM FUNÇÃO DE NÍVEIS DE ADUBAÇÃO NITROGENADA E FORMAS DE APLICAÇÃO DE BORO'
}

\author{
SELMA LOPES DA COSTA², ALMY JUNIOR CORDEIRO DE CARVALHO ${ }^{3}$, PATRÍCIA GOMES DE \\ OLIVEIRA PESSANHA ${ }^{4}$, PEDRO HENRIQUE MONNERAT ${ }^{3}$, CLÁUDIA SALES MARINHO ${ }^{3}$
}

\begin{abstract}
RESUMO - O objetivo deste trabalho foi estudar a influência da adubação com 4 doses de N (0; 100; 200 e $\left.400 \mathrm{~g} \mathrm{planta}^{-1}\right)$, utilizando a uréia, e 3 formas de aplicação do boro (via foliar, via solo e sem B), utilizando o ácido bórico, na produtividade, no número, peso médio, diâmetro e comprimento dos frutos, no crescimento e no diâmetro dos ramos, na percentagem de frutos brocados e rachados. As adubações com boro e nitrogênio aumentaram a produtividade e o número de frutos, não se observando variação no diâmetro e no comprimento dos frutos. Houve variação no peso médio de fruto em função do N. O adubo nitrogenado aumentou o número de flores e de frutos e a porcentagem de frutos. As doses de $\mathrm{N}$ e as formas de aplicação de B não influenciaram as porcentagens de frutos brocados e rachados.
\end{abstract}

Termos de indexação: fruta do conde, nutrição de planta, ácido bórico, uréia.

\section{YIELD OF SUGAR APPLE (Annona squamosa L.) IN FUNCTION OF NITROGEN FERTILIZATION AND FORMS OF APPLICATION OF BORON}

\begin{abstract}
The objective of the research was study the influence of nitrogen fertilization, and three forms of boron application (without boron, boron in the soil and boron on the leaves), on the productivity and the number, average weight, diameter and length of the fruits, as well as on the growth and diameter of the branches, on the percentage of borne. Four doses of $\mathrm{N}(0,100,200$ and 400 $\mathrm{g} \mathrm{plant}^{-1}$ ) were applied. The urea and boric acid were used as $\mathrm{N}$ and B source, respectively. The nitrogen and boron fertilizations enhanced the productivity and the number of fruits. No variation was observed either in the diameter or length of fruits. The average fruit weight varied in function of the $\mathrm{N}$. The nitrogen fertilization increased the fruit yield. Neither nitrogen fertilization nor the method of $\mathrm{B}$ application affected the percentages of borne and split fruits.
\end{abstract}

Index terms: sugar apple, plant nutrition, boric acid, urea.

\section{INTRODUÇÃO}

A região Norte-Noroeste Fluminense, marcada pelo cultivo intensivo e extensivo da cana-de-açúcar, apresenta uma forte vocação para a fruticultura. Sua localização próxima aos grandes centros consumidores (Rio de Janeiro, Vitória, Belo Horizonte e São Paulo) facilita a comercialização e tende a incrementar os ganhos com os produtos vendidos.

A cultura da pinha tem sido apontada como uma das fruteiras mais promissoras para a região, devido à sua fácil adaptação edafoclimática e aos elevados preços que seus frutos alcançam no mercado, com conseqüente interesse por parte dos produtores.

Entretanto, até o momento, pouco se conhece sobre tecnologias de produção da pinha, como obtenção de variedades ou cultivares, poda, indução floral, polinização, controle de pragas e doenças, conservação pós-colheita, adubação e nutrição de plantas.

Entre as técnicas culturais mais importantes, está a nutrição das plantas, pois as fruteiras, de modo geral, retiram do solo grande quantidade de nutrientes, e um desbalanço nutricional pode comprometer a produção. Quando se trata de adubação de fruteiras, o nitrogênio (N) e o boro (B) são nutrientes que merecem especial atenção, pois afetam diretamente a produção, a produtividade e a qualidade de frutos, fatores essenciais ao sucesso da cultura.

O nitrogênio é um nutriente bastante estudado, e a sua disponibilidade é um dos fatores que limitam o crescimento e a produtividade das plantas, pois é requerido em todas as fases do desenvolvimento vegetal (Fernandes e Rossielo, 1995, Maschner, 1995). Plantas deficientes em $\mathrm{N}$ apresentam-se amareladas e com crescimento reduzido. A clorose desenvolve-se primeiro nas folhas mais velhas, com as mais novas permanecendo verdes. Em casos de deficiências severas, as folhas adquirem coloração marrom e morrem (Raij, 1991).

Na Índia, Sadhu e Ghosh (1976), citados por Silva e Silva (1997), trabalhando com três doses de nitrogênio em solo arenoso, constataram que plantas de pinha deficientes em $\mathrm{N}$ apresentaram paralisação do crescimento, não havendo emissão de ramos. As folhas tornaram-se significativamente menores, e de

1 (Trabalho 151/2001). Recebido: 24/09/2001. Aceito para publicação: 04/06/2002. Parte da Tese de Mestrado do $1^{\circ}$ autor apresentada a UENF/ CCTA, Campos dos Goytacazes-RJ. Apoio Financeiro: FAPERJ

2 Eng ${ }^{0}$ Agr ${ }^{\circ}$, M.Sc., Campos, RJ, UENF/CCTA/LFIT, CEP 28015-620, Fone: 222726 1426. E-mail: selmalc@uenf.br

3 Eng ${ }^{\underline{0}}$ Agr ${ }^{\circ}$, D.Sc., Professores, UENF/CCTA/LFIT, Campos, RJ, CEP 28015-620, Fone: 222726 1426, E-mail: almy@uenf.br

4 Estudante do $8^{\circ}$ período do Curso de Agronomia, UENF/CCTA, Campos, RJ, CEP 28015-620. 
tonalidade verde-pálida amarelada. Surgiram manchas de coloração ferruginosa nas folhas inferiores e houve abscisão precoce das mesmas. A formação de gemas florais foi severamente afetada, e o alto nível de $\mathrm{N}$ antecipou o florescimento em 10 dias, enquanto baixo nível desse nutriente retardou o processo de floração em mais de dois meses.

Diferente do nitrogênio, o boro tem sido pouco estudado, apesar de sua deficiência trazer grandes prejuízos à produção e qualidade do produto. O suprimento adequado deste nutriente para as plantas é essencial para o desenvolvimento normal de frutos e sementes (Woods, 1994) e, segundo Maschner (1995), o número de sementes e ou frutos por planta podem, também, ser diretamente afetados pelo ineficiente suprimento mineral com B, o qual influencia na germinação do tubo polínico.

De acordo com Tanada (1995) e Yamada (2000), a carência de $\mathrm{B}$ paralisa o crescimento dos tecidos meristemáticos das folhas e raízes, devido à interrupção da divisão celular. Woods (1994) relata que as anormalidades encontradas nos meristemas apicais podem ser um efeito secundário do dano ao tecido vascular pela deficiência de B.

Pavan (1997), trabalhando com macieira, variedade Gala, estudou o efeito da aplicação de boro no solo e constatou que o aumento do teor de B no solo depende do tipo de fertilizante e da época de amostragem após a aplicação. A fonte de ácido bórico foi superior ao bórax, principalmente no primeiro ano após a aplicação no solo, devido à maior solubilidade da primeira fonte de $\mathrm{B}$ em relação à segunda. A aplicação de $\mathrm{B}$ no solo aumentou os teores deste elemento nas folhas da macieira, dependendo da fonte e da época de amostragem. Os máximos aumentos de $\mathrm{B}$ nas folhas ocorreram durante os dois primeiros anos, diminuindo nos anos seguintes. Como esperado, devido à sua maior solubilidade, o ácido bórico foi mais efetivo que o bórax no primeiro ano, com tendências de igualar-se a partir do segundo ano. A aplicação de B no solo aumentou rapidamente as concentrações no solo e nas folhas, resultando em aumento na produção de frutos.

O objetivo deste trabalho foi estudar a influência da aplicação de nitrogênio e métodos de aplicação de boro no florescimento, vingamento de flores, desenvolvimento de fruto e na produtividade de plantas de pinha.

\section{MATERIAL E MÉTODOS}

O experimento foi conduzido numa propriedade situada na localidade de Praça João Pessoa, no município de São Francisco de Itabapoana-RJ, Latitude $21^{\circ} 19$ ' $22^{\prime \prime}$ e Longitude $41^{\circ}$ 07' 42", em uma área cultivada compreendendo um ha com plantas de pinha com 4 anos de idade, num solo do tipo ARGISSOLO AMARELO distrófico, desenvolvido a partir de sedimentos terciários (Manzatto, 1998). Os resultados da análise química do solo, na camada de 0 a $20 \mathrm{~cm}$ de profundidade, são apresentados no Quadro 1, e as condições climáticas observadas no período de condução do experimento estão apresentadas no Quadro 2.

O delineamento experimental adotado foi o de blocos casualizados, em esquema fatorial 4 × 3 ( 4 doses de $\mathrm{N}$ e 3 formas de aplicação de boro), com três repetições. As parcelas foram constituídas por 3 fileiras com 4 plantas, em espaçamento de $4 \mathrm{x}$ 4 metros, sendo que as 2 plantas centrais foram consideradas úteis, equivalendo a uma área experimental de $32 \mathrm{~m}^{2}$ por parcela e 432 plantas em área total.

Em julho de 2000, efetuou-se uma poda de produção, e as plantas receberam uma adubação com $250 \mathrm{~g}$ de superfosfato simples, $100 \mathrm{~g}$ de cloreto de potássio e $20 \mathrm{~g}$ de sulfato de zinco.

Os tratamentos constaram de 4 doses de nitrogênio $\left(0 ; 100 ; 200\right.$ e 400 g planta $^{-1}$ de N), utilizando-se de uréia como fonte de $\mathrm{N}$, sendo que cada dose foi parcelada em 4 aplicações mensais, de agosto a novembro de 2000; e de 3 formas de aplicação de boro, sendo a aplicação de $40 \mathrm{~g}$ de ácido bórico no solo (parcelada em quatro vezes iguais), pulverizações foliares mensais, de agosto a novembro, de uma solução contendo $0,25 \mathrm{~g}$ de ácido bórico por $100 \mathrm{~L}$ de água e um tratamento sem aplicação de boro.

Durante a condução do experimento, as plantas receberam uma dose fixa de $100 \mathrm{~g}$ de cloreto de potássio em agosto e setembro e $167 \mathrm{~g}$ em outubro e novembro. A plantas foram irrigadas a partir de setembro com um sistema de irrigação por microaspersão, utilizando-se de um microaspersor para 2 plantas.

Além da poda de produção realizada em julho de 2000, as plantas receberam os tratos culturais que se fizeram necessários, tais como, controle de plantas daninhas e tratamentos fitossanitários, com aplicação de DipterexÒ e FolicurÒ para controle da broca-do-fruto (Cerconota anonella) e antracnose (Colletotrichum gloesporioides), respectivamente. Também foram feitas a catação manual e a eliminação com enterrio ou queima dos frutos brocados.

Em setembro e outubro de 2000, foram contadas todas as flores emitidas, para a determinação do número médio de flores/planta, e 60 dias após a primeira contagem de flores (ocasião do máximo de florescimento), contaram-se todos os frutos/planta para a determinação da porcentagem de vingamento de frutos em função dos tratamentos aplicados.

Os efeitos dos tratamentos sobre a produtividade, peso médio, diâmetro, comprimento médio e número de frutos ha ${ }^{-1}$ foram observados em frutos colhidos da parcela útil, pelo menos duas vezes por semana, no período de produção (dezembro de 2000 e janeiro de 2001).

A porcentagem de frutos brocados e rachados foi obtida contando-se, ainda nas plantas, os frutos com esses problemas, os quais eram então retirados e considerados frutos nãocomerciais.

Foram realizadas análises de variância para os parâmetros quantificados e a diferença entre as médias dos tratamentos foi verificada pelo teste de Tukey, ao nível de 5\% de probabilidade, para o Fator Boro, e pelo teste de regressão polinomial, para o Fator Nitrogênio.

\section{RESULTADOS E DISCUSSÃO}

As aplicações de B, tanto no solo quanto nas folhas, provocaram aumento na produtividade e no número de frutos da pinha (Quadro 3). Resultado semelhante foi encontrado por Pavan (1997) quando aplicações de B no solo também elevaram a produtividade de frutos de macieira.

Observou-se efeito quadrático do incremento nas doses de $\mathrm{N}$ sobre o número de frutos e a produtividade. O maior

Rev. Bras. Frutic., Jaboticabal - SP, v. 24, n. 2, p. 543-546, agosto 2002 
número de frutos, 22.365 frutos ha ${ }^{-1}$, e a maior produtividade, $6.059 \mathrm{~kg} \mathrm{ha}^{-1}$, foram obtidos com as doses de 234,7 e 240,1 g planta $^{-1}$ de N, respectivamente (Figura 1).

As diferentes formas de aplicação de B não influenciaram o peso médio, o comprimento e o diâmetro de frutos, cujas médias foram $274 \mathrm{~g}, 73,3 \mathrm{~mm}$ e 81,5 mm, respectivamente (Quadro 4). Com relação às doses de $\mathrm{N}$ aplicadas, não se verificou diferença para o comprimento, o diâmetro e o peso médio de frutos. Apesar de não terem sido verificadas diferenças significativas, é provável que o maior peso médio de frutos observado no tratamento, sem adição de boro, tenha sido provocado pelo menor número de frutos observado nestes tratamentos (Quadro 4).

Não houve diferença entre os tratamentos com B e N para a porcentagem de frutos brocados e rachados. Observaram-se, em média, 4,5\% de frutos brocados e $17,8 \%$ de frutos rachados.

As diferentes formas de aplicação de B não influencia- ram no número de flores por planta, obtido no máximo florescimento, e no número de frutos por planta, 60 dias após o máximo florescimento, cujas médias foram, respectivamente, 416 e 62,9 (Quadro 4). Observou-se elevação na percentagem de frutos vingados quando se comparam os tratamentos com B foliar e aquele em que não foi aplicado o nutriente (Quadro 4).

Verificou-se que o incremento nas doses de $\mathrm{N}$ influenciou o número de flores por planta e o número de frutos por planta, quantificados 60 dias após o florescimento (Figura 2). O maior número de flores por planta, 516, e o maior número de frutos por planta, 60 dias após o florescimento, 95, foram obtidos com a aplicação de 251 e $243 \mathrm{~g}$ de N por planta, respectivamente (Figura 2). A adubação nitrogenada influenciou na percentagem de frutos vingados $\left(\mathrm{Y}=12,5+0,0282 \mathrm{~N}-0,00005352 \mathrm{~N}^{2} \mathrm{R}^{2}=\right.$ $\left.0,70^{*}\right), 60$ dias após o máximo de florescimento, quando a maior percentagem de vingamento de frutos $(19,9 \%)$ foi obtida com a dose de 263,4 g planta $^{-1}$ de $\mathrm{N}$.

TABELA 1 - Características químicas das amostras de solo da área de instalação do experimento.

\begin{tabular}{|c|c|c|c|c|c|c|c|c|c|c|}
\hline \multirow{2}{*}{$\underset{\mathrm{H}_{2} \mathrm{O}}{\mathrm{H} \text { e m }}$} & $\bar{P}$ & $\mathrm{~K}$ & $\mathrm{C} \mathrm{a}$ & $\mathrm{Mg}$ & A 1 & $\overline{\mathrm{H}+\mathrm{A} 1}$ & $\mathrm{~N} \mathrm{a}$ & $\bar{C}$ & $\mathrm{M.O}$ & C.E. \\
\hline & $\mathrm{m} \mathrm{g} \mathrm{d} \mathrm{m}^{-}$ & \multicolumn{6}{|c|}{$\mathrm{cmol} \mathrm{l}_{\mathrm{c}} \mathrm{dm}$} & $\mathrm{g} \mathrm{d} \mathrm{m}^{-3}$ & $\mathrm{~g} \mathrm{~d} \mathrm{~m}^{-3}$ & $\mu \mathrm{S} \mathrm{c} \mathrm{m^{-1 }}$ \\
\hline 6.30 & 29,20 & 0,29 & 1,76 & 0,40 & 0,03 & 1,17 & 0,05 & 7,20 & 12,44 & 882,3 \\
\hline S.B & $\mathrm{T}$ & $\mathrm{t}$ & $\mathrm{m}$ & & $\bar{V}$ & $\mathrm{Fe}$ & $\overline{\mathrm{Cu}}$ & $\mathrm{Zn}$ & $M n$ & $B$ \\
\hline \multicolumn{3}{|c|}{$\mathrm{cmol} l_{\mathrm{c}} \mathrm{d} \mathrm{m}$} & \multicolumn{3}{|c|}{$\%$} & \multicolumn{5}{|c|}{$\mathrm{mg} \mathrm{d} \mathrm{m}$} \\
\hline 2,50 & 3,67 & 2,53 & 1,19 & &, 12 & 10,8 & 0,40 & 1,30 & 28,9 & 0,83 \\
\hline
\end{tabular}

Extratores: $\mathrm{Ca}, \mathrm{Mg}$ e $\mathrm{Al}=\mathrm{KCl} 1 \mathrm{~mol} \mathrm{~L}^{-1}$; P e K = Mehlich-1; $\mathrm{H}+\mathrm{Al}=$ acetato de cálcio 0,5 mol L-1 a pH 7,0; Mn, Fe, Zn e Cu = DTPA; B = água quente Laboratório de Análises de Solo da UFRRJ, Câmpus Leonel Miranda, C. dos Goytacazes - RJ.

TABELA 2 - Características climáticas observadas no ano de 2000, no local de instalação do experimento.

\begin{tabular}{|c|c|c|c|c|}
\hline M ês & $\begin{array}{l}\text { Precipitação } \\
\left(\mathrm{m} \mathrm{m} \mathrm{mês}^{-1}\right)\end{array}$ & $\begin{array}{c}\text { Um idade relativa } \\
(\%)\end{array}$ & $\begin{array}{c}\text { Temperatura do ar } \\
\left({ }^{\circ} \mathrm{C}\right)\end{array}$ & $\begin{array}{c}\text { E T o } \\
\left(\mathrm{m} \mathrm{m} \mathrm{m} \mathrm{ês}{ }^{-1}\right)\end{array}$ \\
\hline Janeiro & 129,50 & 79,34 & 25,13 & 139,3 \\
\hline Fevereiro & 75,00 & 79,11 & 25,34 & 130,6 \\
\hline $\mathrm{M}$ arço & 22,25 & 78,66 & 24,80 & 113,7 \\
\hline A bril & 65,65 & 79,00 & 23,25 & 87,8 \\
\hline M aio & 0,00 & 77,65 & 20,94 & 79,5 \\
\hline Junho & 0,00 & 77,87 & 20,42 & 75,6 \\
\hline $\mathrm{Ju} 1 \mathrm{ho}$ & 13,75 & 76,00 & 19,30 & 79,2 \\
\hline A gosto & 17,75 & 76,00 & 20,30 & 53,0 \\
\hline Setem bro & 53,75 & 81,70 & 21,00 & 64,0 \\
\hline Outubro & 77,70 & 77,10 & 24,00 & 140,8 \\
\hline Novem bro & 174,30 & 80,70 & 23,80 & 125,1 \\
\hline D e z e m bro & 81,70 & 77,38 & 25,17 & 153,30 \\
\hline
\end{tabular}

Local: São Francisco do Itabapoana - RJ. Latitude: $21^{\circ} 19^{\prime} 22^{\prime \prime}$ Sul e Longitude: $41^{\circ} 07^{\prime} 42^{\prime}$ ' Oeste

TABELA 3 - Produtividade e número de frutos/ha na cultura da pinha, em função da adubação nitrogenada e da forma de aplicação de boro.

\begin{tabular}{c|c|c|c|c|c|c|c|c}
\hline \multirow{2}{*}{$\begin{array}{c}\mathrm{N} \\
\text { g planta }\end{array}{ }^{-1}$} & \multicolumn{3}{|c|}{ Produtividade $\left(\mathrm{kg} \mathrm{ha}^{-1}\right)$} & \multicolumn{4}{c}{$\mathrm{N}^{\mathbf{0}}$ de frutos (ha) } \\
\cline { 2 - 9 } & Sem B & B solo & B foliar & Média & Sem B & B solo & B foliar & Média \\
\hline 0 & 4.380 & 4.045 & 4.517 & 4.314 & 16.771 & 16.030 & 18.204 & 17.002 \\
100 & 4.728 & 5.966 & 5.615 & 5.436 & 13.958 & 23.958 & 18.497 & 18.805 \\
200 & 5.578 & 5.421 & 7.065 & 6.021 & 21.146 & 21.667 & 27.500 & 23.438 \\
400 & 4.912 & 5.161 & 5.745 & 5.272 & 18.273 & 18.854 & 20.521 & 19.216 \\
\hline Média & $4.899 \mathrm{~b}$ & $5.148 \mathrm{ab}$ & $5.735 \mathrm{a}$ & 5.261 & $17.537 \mathrm{~b}$ & $20.127 \mathrm{ab}$ & $21.181 \mathrm{a}$ & 19.615 \\
\hline (CV\%) & \multicolumn{3}{|c|}{13,3} & \multicolumn{5}{c}{17,1} \\
\hline
\end{tabular}

Médias seguidas pela mesma letra, na linha, não diferem, pelo teste de Tukey, ao nível de 5\% de probabilidade. 
TABELA 4 - Peso médio, diâmetro e comprimento de frutos, $\mathrm{N}^{\circ}$ de flores/planta no máximo florescimento, $\mathrm{N}^{\circ}$ de frutinhos/planta, 60 dias após o máximo florescimento, e \% de vingamento de frutos na cultura da pinha, em função da forma de aplicação de boro.

\begin{tabular}{|c|c|c|c|c|c|c|}
\hline Tratamento & $\begin{array}{l}\text { Peso de } \\
\text { fruto } \\
(\mathrm{g})\end{array}$ & $\begin{array}{l}\text { Diâmetro } \\
\text { de fruto } \\
(\mathrm{mm})\end{array}$ & $\begin{array}{l}\text { Comprimento } \\
\text { de fruto } \\
(\mathrm{mm})\end{array}$ & $\begin{array}{c}\mathrm{N}^{\circ} \text { de flores/planta } \\
\text { no máximo } \\
\text { florescimento }\end{array}$ & $\begin{array}{c}\mathrm{N}^{\circ} \text { de frutinhos/planta } \\
60 \text { dias após } \\
\text { florescimento }\end{array}$ & $\begin{array}{c}\text { \% vingamento de } \\
\text { frutos } 60 \text { após } \\
\text { máximo } \\
\text { florescimento }\end{array}$ \\
\hline $\begin{array}{l}\text { Sem B } \\
\text { B solo } \\
\text { B foliar }\end{array}$ & $\begin{array}{l}289 \mathrm{a} \\
259 \mathrm{a} \\
274 \mathrm{a}\end{array}$ & $\begin{array}{l}82,8 \mathrm{a} \\
80,0 \mathrm{a} \\
81,7 \mathrm{a}\end{array}$ & $\begin{array}{l}74,4 \text { a } \\
72,3 \text { a } \\
73,2 \text { a }\end{array}$ & $\begin{array}{l}428 \mathrm{a} \\
410 \mathrm{a} \\
410 \mathrm{a}\end{array}$ & $\begin{array}{l}58,5 \text { a } \\
62,3 \text { a } \\
68,0 \text { a }\end{array}$ & $\begin{array}{l}13,7 \mathrm{~b} \\
15,1 \mathrm{ab} \\
16,6 \mathrm{a}\end{array}$ \\
\hline Média & 274 & 81,5 & 73,3 & 416 & 62,9 & 15,1 \\
\hline $\mathrm{CV}(\%)$ & 11,7 & 6,12 & 7,64 & 23,5 & 26,0 & 10,2 \\
\hline
\end{tabular}

Médias seguidas pela mesma letra, na coluna, não diferem, pelo teste de Tukey, ao nível de 5\% de probabilidade.

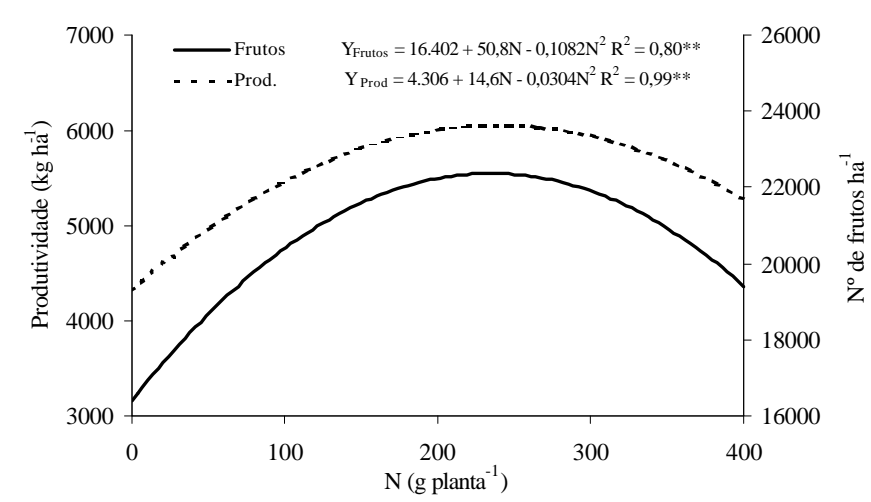

FIGURA 1 - Produtividade, em kg ha ${ }^{-1}$, e número de frutos ha' ${ }^{-1}$, na cultura da pinha, em função de doses de nitrogênio, em g planta-1.

\section{CONCLUSÕES}

1. As adubações com nitrogênio e com boro aumentaram o número de frutos e a produtividade das plantas.

2. A adubação nitrogenada aumentou o número de flores e o vingamento de frutos.

3. A aplicação foliar de boro elevou o vingamento dos frutos.

\section{REFERÊNCIAS BIBLIOGRÁFICAS}

FERNANDES, M.S.; ROSSIELLO, R.O.P. Mineral Nitrogen in Plant Physiology and Plant Nutrition. Critical Reviews in Plant Sciences, Boca Raton, v.14, n.2, p.111-148, 1995.

MAnZATTO, C.V. Pedogênese Topossequencial de Solos desenvolvidos de Sedimentos do Terciário do Norte Fluminense: Um Subsídio ao Manejo Agrícola Racional, 1998. 148f. Tese (Doutorado em Produção Vegetal) - Universidade Estadual do Norte Fluminense, Campos dos Goytacazes, 1998.

MARSCHNER, H. Mineral nutrition of higher plants. London: Academic Press, 1995. 889p.

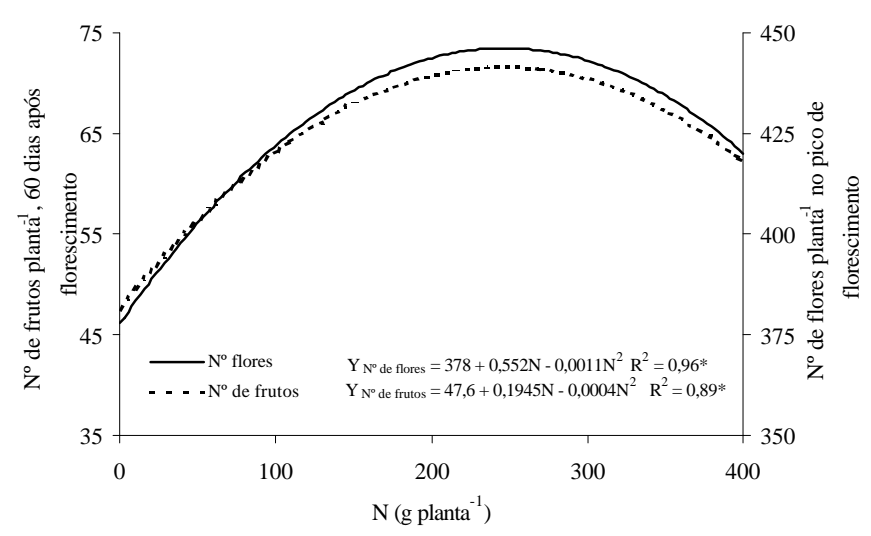

FIGURA 2 - Número de flores por planta no pico de florescimento e número de frutos por planta, 60 dias após o pico do florescimento, na cultura da pinha, em função das doses de nitrogênio, em g planta ${ }^{-1}$.

PAVAN, M. Respostas da macieira à aplicação de boro no solo. Arquivo Biológico Tecnológico, Campinas, v.40, n.2, p.419-424, 1997.

RAIJ, B. Van. Fertilidade do solo e adubação. Piracicaba: Ceres/ POTAFOS, 1991.343p.

SILVA, A.Q.; SILVA, H. Nutrição e Adubação de Anonáceas. In: SÃO JOSÉ, A.R.; SOUZA, I.V.B.; MORAIS, O.M.; REBOUÇAS, T.N.H. Anonáceas, produção e mercado (Pinha, Graviola, Atemóia e Cherimólia). Vitória da Conquista (BA): DFZ/UESB, 1997. p.118-137.

TANADA, T. Boron as a transducer in some physiological processes of plants. Journal of Plant Nutrition, Monticello, v.18, n.9, p.1743-1750, 1995.

WOODS, W.G. An Introduction to Boron: History, Sources, Uses, and Chemistry. Environmental Health Perspectives; Washington, v.102, n.7, p.5-11, 1994.

YAMADA, T. Boro: será que estamos aplicando a dose suficiente para o adequado desenvolvimento das plantas? Informações Agronômicas-POTAFOS, n.90, p.1-5, 2000. 